\title{
Practical Applications of Astropy
}

\author{
David Shupe ${ }^{\ddagger *}$, Frank Masci, Russ Laher ${ }^{\ddagger}$, Ben Rusholme* ${ }^{\ddagger}$, Lee Armus \\ https://youtu.be/2GTLkH5sfJc
}

\begin{abstract}
Packages developed under the auspices of the Astropy Project ([ART ${ }^{+}$13], [TPS $\left.\left.{ }^{+} 18\right]\right)$ address many common problems faced by astronomers in their computational projects. In this paper we describe how capabilities provided by Astropy have been employed in two current projects. The data system for the Zwicky Transient Facility processes a terabyte of image data every night, with a lights-out automated pipeline that produces difference images about ten minutes after the receipt of every exposure. Astropy is used extensively in the astrometry and light-curve-generation modules, making especially heavy use of FITS header manipulation, table $\mathrm{I} / \mathrm{O}$, and coordinate conversion and matching. The second project is a web application made with Plotly Dash for proposal studies for the Origins Space Telescope. The astropy.cosmology module provided easy redshifting of our template galaxy spectrum, and astropy.units enabled the porting of an instrument sensitivity function to Python, with verification that a very complex combination of units resulted in a dimensionless signal-to-noise value.
\end{abstract}

Index Terms-astronomy, data processing

\section{Introduction}

The Astropy Project is a community-driven effort to provide both a core Python package of functionality commonly used by astronomers, and an extended ecosystem of interoperable packages, with high standards for documentation and testing ([ART $\left.{ }^{+} 13\right]$, $\left.\left[\mathrm{TPS}^{+} 18\right]\right)$. The astropy core package includes subpackages for representing and manipulating space and time coordinates; I/O for astronomical file formats; world coordinate systems in images (e.g. converting between celestial coordinates and image pixels); cosmological calculations; and manipulating numerical quantities with units. Most astronomers using the astropy core package use it for interactive analyses. In this paper, we highlight the importance of astropy in two production environments: the data system for the Zwicky Transient Facility (ZTF), and a web application for the proposed Origins Space Telescope.

\section{The ZTF Project}

The Zwicky Transient Facility (ZTF) is a new robotic survey now underway, using the 48-inch Samuel Oschin Telescope at Palomar Observatory in southern California. This telescope was originally constructed to take images with photographic plates, in large part to provide targets for the 200-inch Hale Telescope at the same observatory. The ZTF camera fills the focal plane of the 48 -inch

* Corresponding author: shupe@ipac.caltech.edu \$Caltech/IPAC

Copyright $(92018$ David Shupe et al. This is an open-access article distributed under the terms of the Creative Commons Attribution License, which permits unrestricted use, distribution, and reproduction in any medium, provided the original author and source are credited. telescope with sixteen $6 \mathrm{k} \times 6 \mathrm{k}$ charge-coupled devices (CCDs) with an active detector area of 47 square degrees (Dekany et al in prep; $\left.\left[\mathrm{DSB}^{+}{ }^{16}\right]\right)$. ZTF is conducting a fast, wide-area timedomain survey (Bellm et al in prep) designed to discover fast, young and rare flux transients; counterparts to gravitational wave sources; low-redshift Type Ia supernovae for cosmology; variable stars and eclipsing binaries; and moving objects in our Solar System such as asteroids (Graham et al in prep). The entire sky visible to Palomar can be imaged each night to declinations above -30 degrees. The survey began in March 2018 and will continue for three years. Figure 1 shows a field-of-view comparison of ZTF with its predecessor at Palomar, the Palomar Transient Factory (PTF; [LKD $\left.{ }^{+} 09\right]$ ), and the forthcoming Large Synoptic Survey Telescope (LSST).

A typical night of ZTF observations includes about 750 exposures totaling about 1 Terabyte of image data when uncompressed. Each quadrant of the CCDs is processed separately for a total of about 55,000 calibrated science images per night. Depending on sky location, 0.5 to 1 billion individual source measurements are extracted per night. The ZTF data system (Masci et al. 2018, in review, $\left.\left[\mathrm{LMG}^{+} 18\right]\right)$ is operated by the IPAC data center on the Caltech campus. Within a few minutes of receipt of an exposure at IPAC, a real-time image subtraction pipeline outputs alert packets of potential transient objects, at rates already nearing 1 million per night. Alert packets from the public portion of the survey have just become available ${ }^{1}$, along with a repository of the schema and code examples ${ }^{2}$.

The data system is mostly scripted in Perl, with job management relying on a Postgres database. A cluster of 66 compute nodes handles the processing. Astropy is used in several key components of the pipeline. In the following subsections we outline Astropy use and what we've learned from operational experience.

\section{Improving reliability of the astrometric solver}

Assigning coordinates to ZTF images is challenging for several reasons. The accuracy of the pointing of the boresight (center of the field-of-view) is about 20 arcseconds rms. Atmospheric effects cause image distortions on small scales, and these effects are exacerbated at low elevations. ZTF employs the Scamp astrometric solver from the Astromatics suite ([Ber06]) to match star positions from the Gaia Data Release 1 (DR1) catalog $\left(\left[\mathrm{GPd}^{+} 16\right]\right.$, $\left.\left[\mathrm{GBV}^{+} 16\right]\right)$ and ultimately fit a 4th-order polynomial to the image distortions. Scamp is written in $\mathrm{C}$ and requires inputs in a very

1. https://ztf.uw.edu/alerts/public

2. https://github.com/ZwickyTransientFacility/ztf-avro-alert 


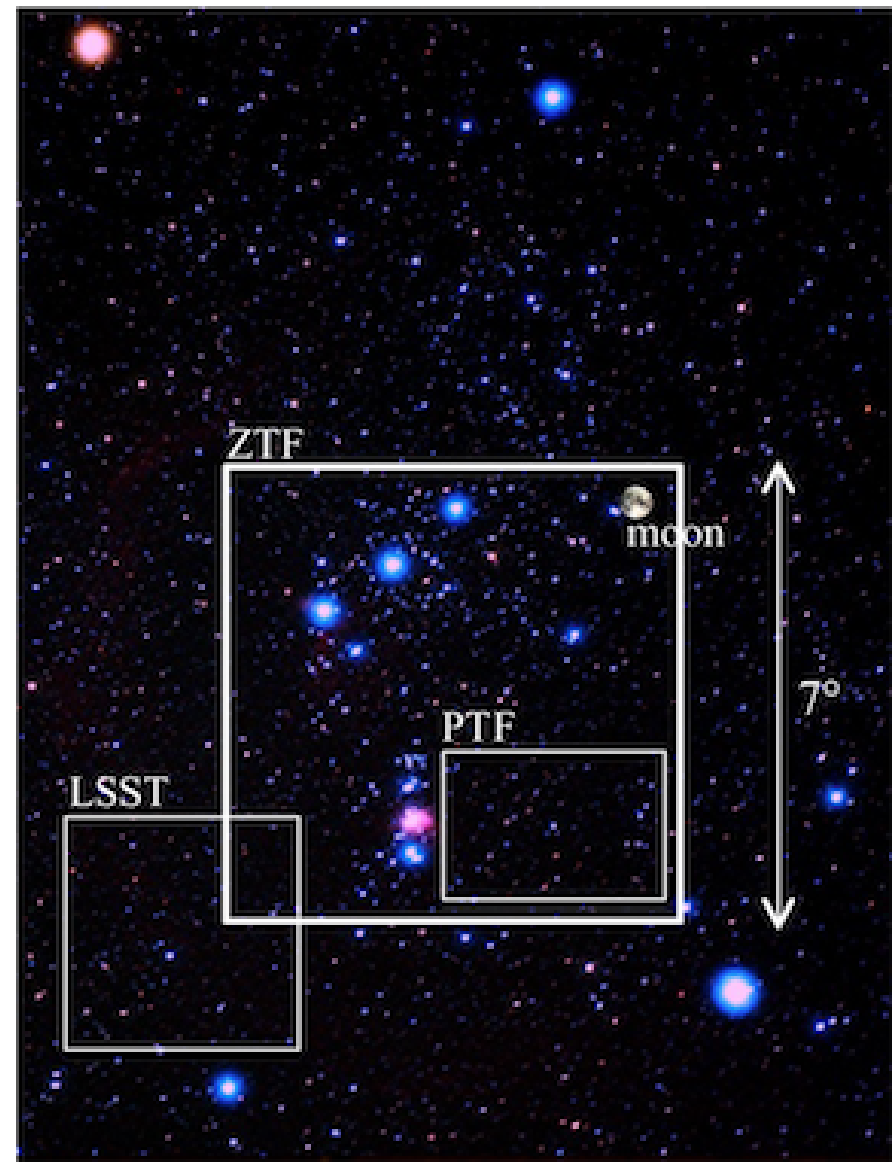

Fig. 1: Field of view of the ZTF camera, compared to the predecessor Palomar Transient Factory (PTF) camera, and the forthcoming Large Synoptic Survey Telescope (LSST). The background image shows the Orion constellation

specialized format. We have developed a procedure that has significantly reduced the rate of incorrect solutions in crowded fields, by providing Scamp with an accurate starting point (see Figure 2).

Scamp requires both the input catalog of detections and the reference catalog to be provided in LDAC (Leiden Data Analysis Center $)^{3}$ FITS format. This format consists of header information encoded in a binary format in a table extension, followed by another table extension of detections. Recent versions of Scamp will start from a prior World Coordinate System (WCS; [CG02]) solution provided to the program. Providing a distortion prior derived from many observations makes it much easier for Scamp to converge on the global minimum, i.e. the correct distortion solution. Our efforts to include the WCS in the LDAC file of detections using astropy.io.fits were unsuccessful. However, the WCS information in the LDAC file can be overridden by a text file of header information provided separately to Scamp.

Our distortion prior is constructed from an offline analysis of images taken at high elevations (low airmasses), the same conditions used in the ZTF survey. For selected fields, we set up idealized WCS objects with 1 degree per "pixel":

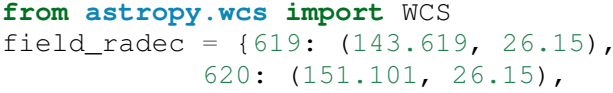

3. https://marvinweb.astro.uni-bonn.de/data_products/THELIWWW/ LDAC/LDAC_concepts.html

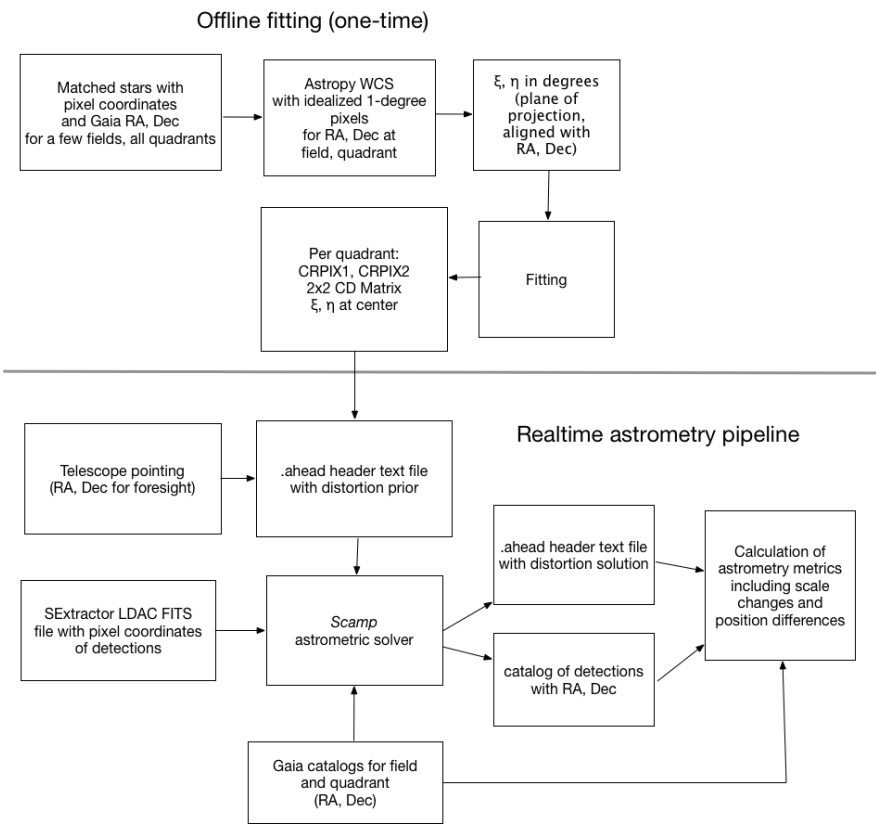

Fig. 2: Processing diagrams for ZTF astrometry. An offline analysis (top) is performed on a few exposures to make a prior model for each of the 64 quadrants in the focal plane. These terms are combined with telescope pointing to make an accurate prior for Scamp in the realtime pipeline (bottom), resulting in a calibrated header text file with the full coordinate solution including distortions, and a catalog of the detected stars with assigned RA and Dec coordinates. These outputs of the astrometric fitting are matched again with the Gaia reference catalog to produce metrics for assessing the quality of the astrometric fit.

$$
\begin{aligned}
& 665:(133.35,33.35), \\
& 667:(149.057,33.35)\}
\end{aligned}
$$

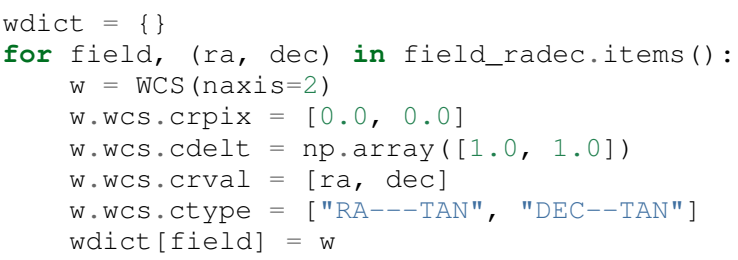

Then when reading in a catalog of sources with positions for each field, we convert the right ascensions and declinations to projection plane coordinates ([CG02]) $\xi, \eta$ in units of degrees in the tangent plane:

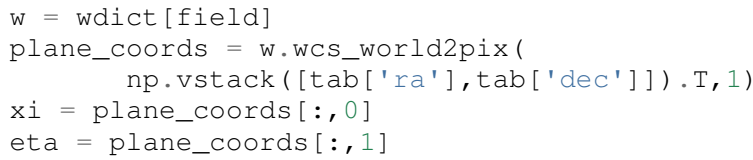

A linear model is fit relating image pixel values to the computed $\xi$ and $\eta$ values, while allowing offsets and linear terms for each exposure and readout channel. This fit yields the CRPIX1 and CRPIX2 values (pixel offsets) from the telescope boresight to each of the 64 readout channels. This linear solution yields residuals of about four arcseconds in magnitude. Then "global" pixel coordinates are constructed and a quadratic fit relating these to $\xi$ and $\eta$ is computed. This second fit is used to find $\xi$ and $\eta$ for the center of each quadrant-image. For each quadrant-image, a linear fit is made to yield the multiplicative terms for pixel scale and rotation (CD-matrix values; [CG02]) for each quadrant. This 
procedure transfers the pointing to the center of each individual quadrant-image.

The CD-matrix, CRPIX1, CRPIX2, and $\xi, \eta$ values for each quadrant are saved to be used by the astrometry pipeline. The parameters are read and inserted into a text file (.ahead file) that initializes Scamp. For each image, a first run of Scamp is made using 'PRE-DISTORTED' mode. This performs pattern-matching of detected stars and reference stars from Gaia DR1. Scamp is allowed only a little freedom to rotate and change scale. A second pass of Scamp skips the pattern-matching and fits a fourth-degree distortion polynomial as part of the output WCS. An essential speed improvement was pre-fetching static copies of the Gaia DR1 catalog and storing these in the LDAC FITS format using astropy.io.fits, in a static area, to be available as static catalogs for Scamp.

\section{Assessing the quality of the astrometric solution}

A problem encountered often in the PTF survey was not being able to readily tell whether a solution output by Scamp was of poor quality. Astrometric problems greatly increase the number of spurious transients produced by image subtraction and later steps of the pipeline and scanning processes. Scamp does output a chisquared statistic. When provided with realistic errors, most good solutions result in a chi-squared statistic of about five. To ensure that the system catches the case of a distortion polynomial that is unconstrained in the corner of an image, we developed a scale check test of the final solution against the distortion prior that we initially provided to Scamp.

First we generate a grid over the detector, and then make pixel coordinates at each grid point:

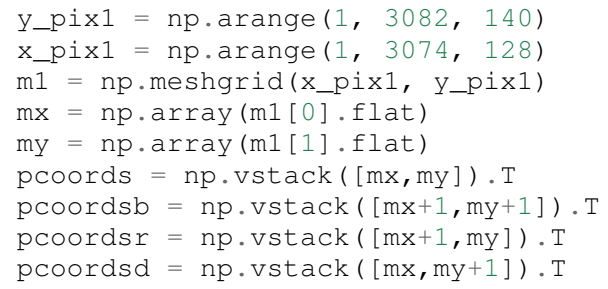

Then using the WCS objects from the prior and from the final solution, we calculate pixel areas:

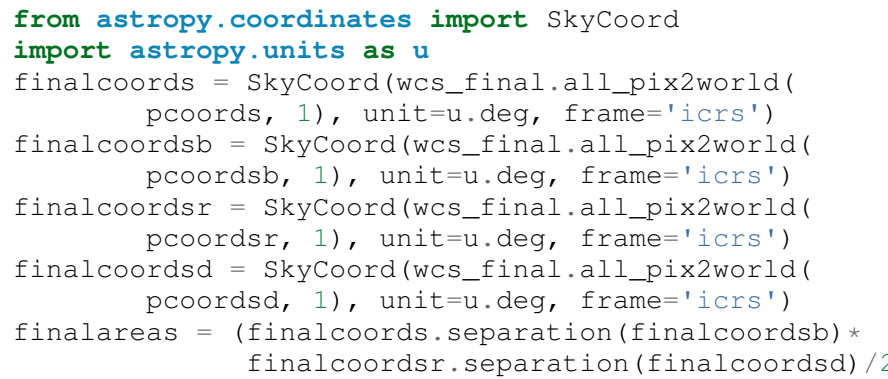

These steps are repeated for the prior. Finally we compute a percentage change in pixel scale:

pctscaledif $=100 *(n p . s q r t(f i n a l a r e a s)$

np.sqrt (priorareas)) /np.sqrt (priorareas)

If the percentage scale difference changes by more than a percent, the image is marked as unusable. Figure 3 shows the mean value of the percentage scale difference for a night of ZTF commissioning exposures, showing the changes follow a model $^{4}$ for differential atmospheric refraction.

4. http://wise-obs.tau.ac.il/ eran/Wise/Util/Refraction.html

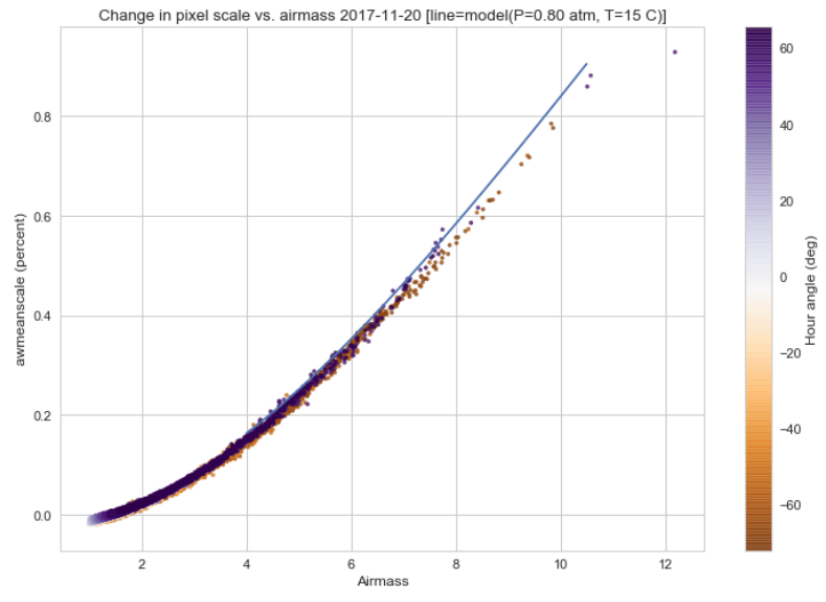

Fig. 3: Mean pixel scale versus airmass for one night of commissioning data. The line shows model points for pressure and temperature appropriate for Palomar Observatory.

A peculiarity for ZTF is that with a field-of-view that is seven degrees on a side, the airmass reported by the telescope control system does not apply well for the outer CCDs. We use an AltAz model to recompute airmass when analyzing metric values for the pixel scale change.

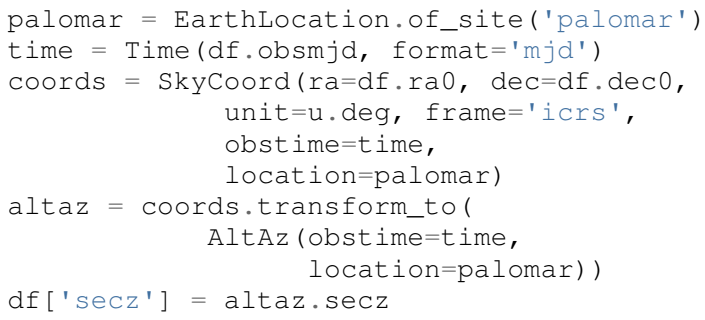

A future update to the astrometry module, now being tested, distorts the CD-matrix along the azimuthal direction and by a magnitude determined from the differential refraction model. The correction is not needed for the main survey and will help find solutions for targets of opportunity at high airmass.

\section{Accounting for light-travel-time in ZTF light curves}

For ZTF, the PSF-fitting photometry that is extracted from every image is periodically combined into matchfiles in HDF5 format. These matchfiles form the basis of the lightcurve service that will be deployed by IPAC's Infrared Science Archive. The matchfiles are also used to provide light curves for variable star studies.

The matchfiles are seeded by PSF-fitting photometry extracted from reference images. The reference images are coadds of between 15 and 40 exposures of a ZTF field. Astropy's SkyCoord class is employed to perform the matching of input sources to reference objects.

Astropy is also used to provide heliocentric Julian dates for each source. The difference between heliocentric Julian date and observed Julian date is the light-travel time difference between the Earth-to-coordinate direction and the Sun-to-coordinate direction. It is computationally prohibitive to compute this time difference for each individual source. Instead, a SkyOffset frame is defined at the maximum coordinate for a field, and then a 9x9 grid is set up on that offset grid. A fit is made of light-travel-time difference as a quadratic function of longitude and latitude in the offset frame. 
This provides an accuracy in the calculation of the heliocentric date that is much less than a ZTF exposure time of 30 seconds.

Since some ZTF fields straddle $\mathrm{RA}=0$, a mean or median of RA yields misleading values. For our nearly-degree-sized fields, we use the maximum values and define an offset frame:

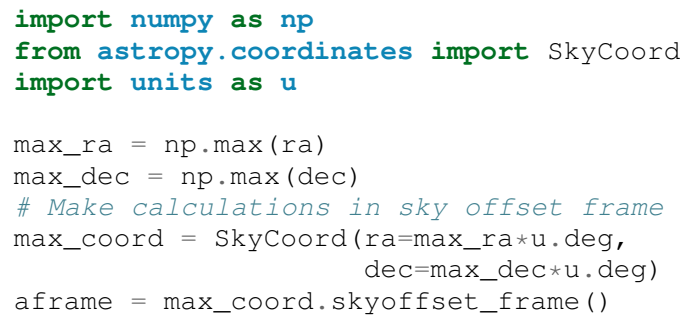

The PSF-fitting catalog coordinates are transformed to the offset frame and a bounding box in that frame is computed:

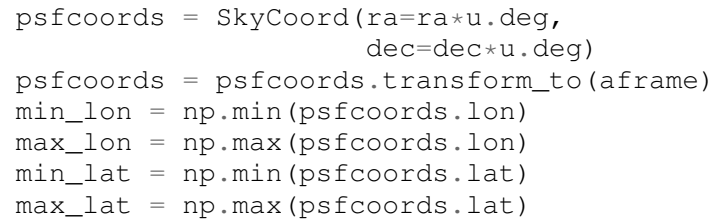

A 9x9 grid is set up in the SkyOffset frame:

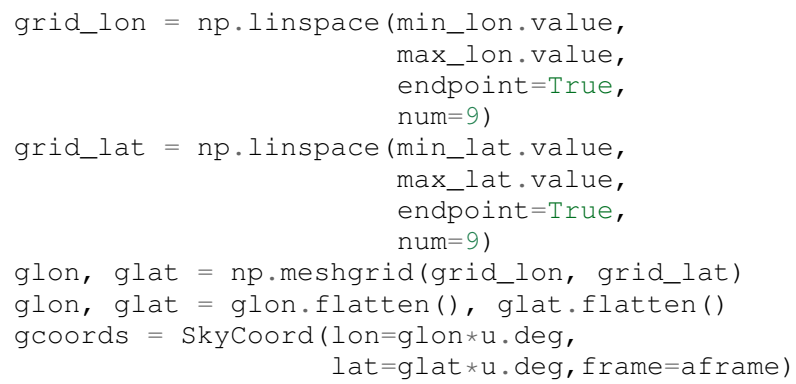

Although coord.EarthLocation.of_site was used in our offline astrometry analysis, its network fetch of coordinates is not reliable for many parallel processes. The hard-coded observatory location is combined with the modified Julian date of the observation to compute light-travel-time over our 9x9 grid:

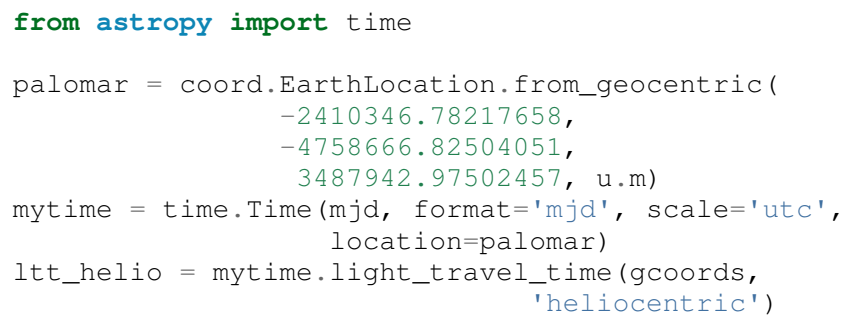

Coefficients for a least-squares fit of a 2-dimensional quadratic surface are computed and applied to our catalog coordinates to yield light-travel-times for each source, and then added to our observed times to result in heliocentric Julian dates:

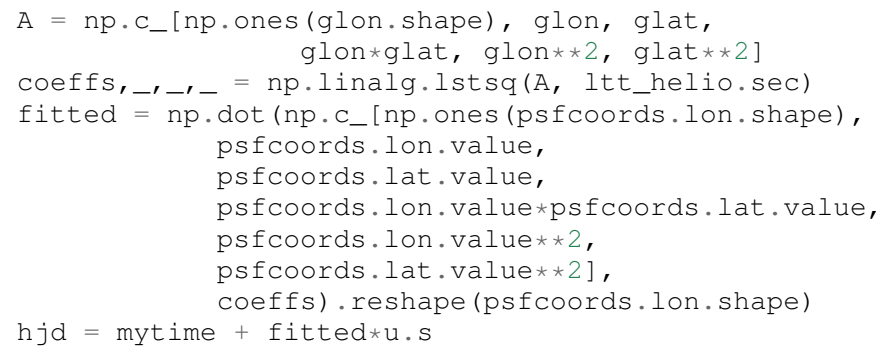

\section{Configuration file issue}

In the course of running the ZTF pipeline in production, we encountered a serious problem caused by the \$HOME/.astropy/config file. This file would randomly corrupt, causing every Astropy import to fail. The cause of the problem was different Astropy versions installed in our Python $2 \& 3$ virtual environments. The configuration file is overwritten every time a different versions of Astropy version is imported. Our pipeline contained a mixture of Python 2 and Python 3 code, running in parallel at enough scale, that a collision would eventually occur. The problem was solved by installing the same version of Astropy in both versions of Python.

\section{Lessons learned from the ZTF experience}

- Python and Astropy worked very well to wrap the Scamp solver and to provide its specialized inputs to make it converge reliably on correct astrometric solutions.

- The key to working with the LDAC format is providing an additional text file header that is easily manipulated with Astropy.

- Astropy.wcs supports TPV distortions since version 1.1, enabling us to compute metrics assessing the quality of the astrometric fits.

- When you have a 7-degree field of view, the elevation, azimuth, and airmass reported by the telescope system lack sufficient precision.

- Elminiate network calls as much as possible, by prefetching the astrometric catalogs, and bypassing astropy.coordinates.EarthLocation.of_site.

- SkyCoord.offset_frame is essential to avoid zero-wrapping problems in celestial coordinates, and is very useful when working on a patch of sky.

- Configuration files can cause problems at scale.

- Technical debt from not converting everything to Python 3 will bite you.

\section{Origins Space Telescope}

The Origins Space Telescope is a space observatory concept under study as part of NASA's astrophysics roadmap. The first design includes a 9-meter primary mirror with all components cooled to less than $6 \mathrm{~K}$, to provide orders of magnitude more sensitivity than previous space infrared missions.

As part of the concept study, a web application has been constructed to showcase the potential of one of the spectroscopic instruments, the Mid-Resolution Survey Spectrometer ([BO18]). The purpose of the application is to allow trade studies of different observational parameters, including the telescope diameter, the exposure time, and the distance to the star or galaxy of interest. Plotly Dash ${ }^{5}$ was chosen as the technology for constructing the project.

Part of the project involved converting a complicated function for instrument sensitivity to Python. The astropy.units and astropy.constants packages made it relatively easy to check the results of the calculation.

Many astronomers are used to working with "magic numbers" that are constants or combinations of constants that we keep in our heads. Here is an example:

5. https://plot.ly/products/dash/ 
freq=double $(2.9979 \mathrm{e} 5 /$ wave $)$; in $\mathrm{GHz}$

$\mathrm{h}=$ double $(6.626 \mathrm{e}-18) ; \mathrm{h}$ in erg / $\mathrm{GHz}$

$\mathrm{c}=$ double $(2.9979 \mathrm{e10}) ; \mathrm{c}$ in $\mathrm{cm} / \mathrm{sec}$

With astropy.units and affiliated packages:

import astropy.constants as const

import astropy.units as $u$

freq $=$ const. c/wave

The noise equivalent flux calculation for the spectrometer depends in part on the numbers of photons (occupation number) coming from the background at a particular wavelength.

$$
\bar{n}=\frac{c^{2} I_{v}}{2 h v^{3}}
$$

where $I_{v}$ is the background intensity in MJy/sr. An assertion in the calculation of occupation number ensures it is dimensionless:

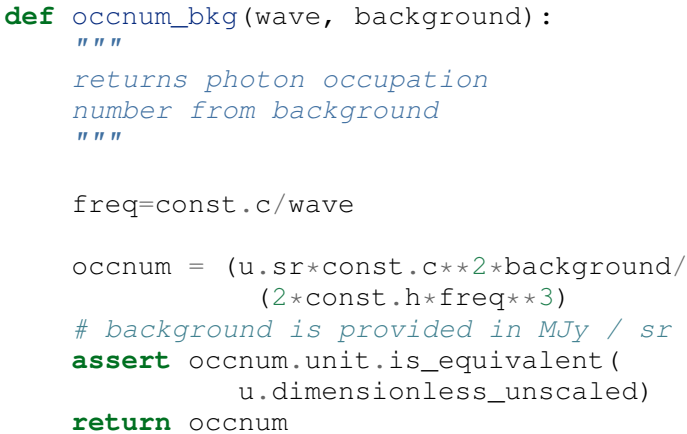

The assertion ensures that the occupation number is dimensionless.

The noise equivalent power for an element in the spectrometer depends the frequency, bandwidth and photon occupation number at that frequency:

$$
N E P=h v \sqrt{\Delta v \bar{n}(\bar{n}+1)}
$$

where the bandwidth $\Delta v=v / R$ and $R$ is the spectrometer resolution. In the instrument sensitivity function, this is implemented with an assertion to check units at an intermediate stage:

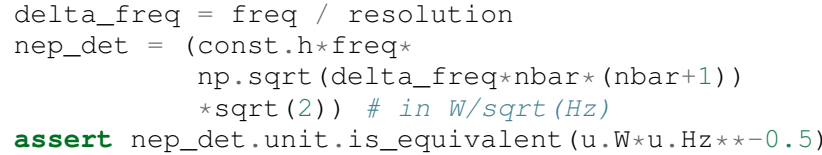

For the extragalactic example in the application, the astropy.cosmology module was used to redshift the spectrum. The Planck 2015 cosmology $\left(\left[\mathrm{PAA}^{+} 16\right]\right)$ is one of the built-in cosmologies in the package. For each user-selected value of redshift, we computed the luminosity distance to scale the flux values of the spectrum.

For re-gridding the wavelength spectrum, we used the pysynphot package (not an astropy package but developed in part by Astropy developers) ([STS13]) to interpolate the redshifted spectrum onto the observed wavelength channels.

The application has been deployed on the Heroku platform ${ }^{6}$. A screenshot of the galaxy spectrum is shown in Figure 4. To ensure good performance when changing parameters, the instrument sensitivity was pre-computed for the lines in the spectra, for different backgrounds and redshifts.

6. https://ost-mrss.herokuapp.com
The astropy.units package is broadly useful outside astronomy; to that end, the unyts package $\left(\left[\mathrm{GZT}^{+} 18\right]\right)$ is a newly-available standalone alternative.

Lessons learned include:

- Using a units package together with assertions at intermediate stages helped to validate a complex instrument sensitivity function.

- However, a units package does not help get factors of $(1+\mathrm{z})$ correct.

- Pre-computing sensitivities for several parameter choices sped up the application.

- The pysynphot functionality for regridding spectra would be useful to break out into a more accessible Astropyaffiliated package.

\section{Conclusions}

This paper highlights the use of Astropy in two production environments: the Zwicky Transient Facility data system, and a web application for the Origins Space Telescope. Astropy's capabilities for manipulating FITS files and image headers, coupled with its coordinate conversion capabilities, helped us implement a scheme to greatly improve the reliability of ZTF astrometry, and provided other conveniences. The astropy.units and astropy.cosmology packages provided essential transformations for the Origins study application. We found that some care needs to be taken with minimizing or eliminating network calls, and with handling configuration files that assume a single package version is in use.

\section{Acknowledgments}

We are grateful to D. Levitan, W. Landry, S. Groom, B. Sesar, J. Surace, E. Bellm, A. Miller, S. Kulkarni, T. Prince and many other contributors to the PTF and ZTF projects. The Origins Space Telescope app includes significant contributions from C.M Bradford, K. Pontopiddan, K. Larson, J. Marshall, and T. DiazSantos.

ZTF is led by the California Institute of Technology, US and includes IPAC, US; the Joint Space-Science Institute (via the University of Maryland, College Park), US; Oskar Klein Centre of the University of Stockholm, Sweden; University of Washington, US; Weizmann Institute of Science, Israel; DESY and Humboldt University of Berlin, Germany; University of Wisconsin at Milwaukee, US; the University System of Taiwan, Taiwan; and Los Alamos National Labora- tory, US; ZTF acknowledges the generous support of the National Science Foundation under AST MSIP Grant No 1440341. The alert distribution service is provided by the DIRAC Institute at the University of Washington. The High Performance Wireless Research \& Education Network (HPWREN; https://hpwren.ucsd.edu) is a project at the University of California, San Diego and the National Science Foundation (grant numbers 0087344 (in 2000), 0426879 (in 2004), and 0944131 (in 2009)).

This work has made use of data from the European Space Agency (ESA) mission Gaia (https://www.cosmos.esa.int/ gaia), processed by the Gaia Data Processing and Analysis Consortium (DPAC, https://www.cosmos.esa.int/web/gaia/dpac/ consortium). Funding for the DPAC has been provided by national institutions, in particular the institutions participating in the Gaia Multilateral Agreement. 


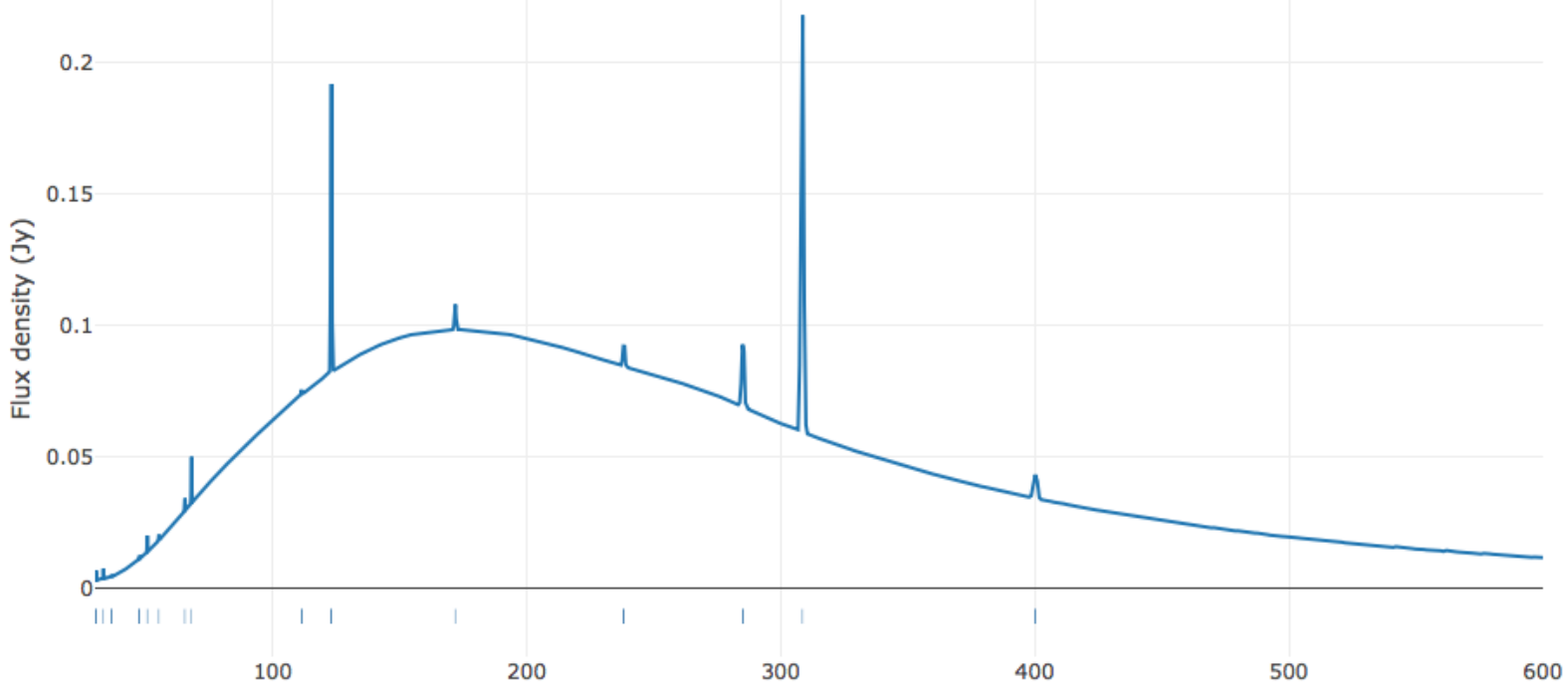

Wavelength Range

Source Spectrum

protoplanetary disk $\odot$ starburst galaxy

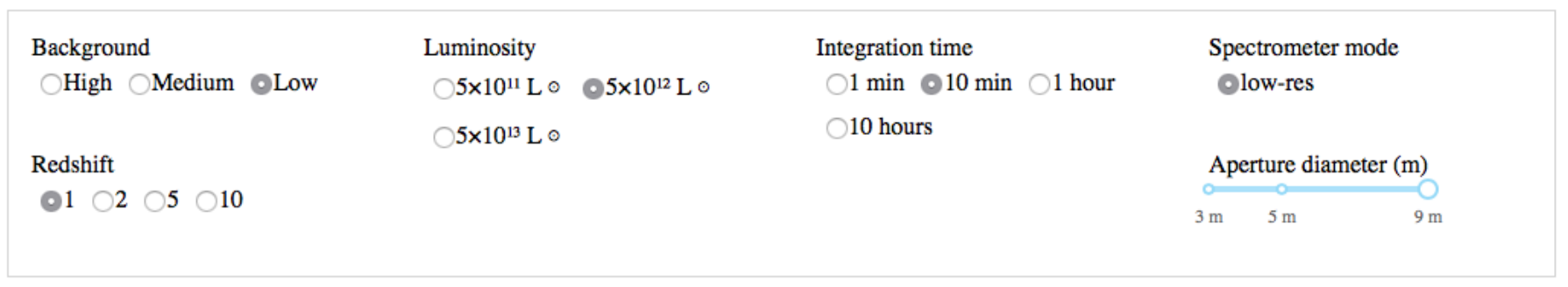

Fig. 4: The web application for the Origins Space Telescope, showing the galaxy spectrum and controls for changing source characteristics and instrument parameters.

\section{REFERENCES}

$\left[\mathrm{ART}^{+} 13\right]$ Astropy Collaboration, Thomas P. Robitaille, Erik J. Tollerud, Perry Greenfield, Michael Droettboom, Erik Bray, Tom Aldcroft, Matt Davis, Adam Ginsburg, Adrian M. Price-Whelan, Wolfgang E. Kerzendorf, Alexander Conley, Neil Crighton, Kyle Barbary, Demitri Muna, Henry Ferguson, Frédéric Grollier, Madhura M. Parikh, Prasanth H. Nair, Hans M. Unther, Christoph Deil, Julien Woillez, Simon Conseil, Roban Kramer, James E. H. Turner, Leo Singer, Ryan Fox, Benjamin A. Weaver, Victor Zabalza, Zachary I. Edwards, K. Azalee Bostroem, D. J. Burke, Andrew R. Casey, Steven M. Crawford, Nadia Dencheva, Justin Ely, Tim Jenness, Kathleen Labrie, Pey Lian Lim, Francesco Pierfederici, Andrew Pontzen, Andy Ptak, Brian Refsdal, Mathieu Servillat, and Ole Streicher. Astropy: A community Python package for astronomy. Astronomy and Astrophysics, 558:A33, October 2013. doi:10.1051/0004-6361/201322068.

[Ber06] E. Bertin. Automatic Astrometric and Photometric Calibration with SCAMP. In C. Gabriel, C. Arviset, D. Ponz, and S. Enrique, editors, Astronomical Data Analysis Software and Systems XV, volume 351 of Astronomical Society of the Pacific Conference Series, page 112, July 2006.

[BO18] Charles Matt Bradford and Origins Space Telescope Study Team. The Medium Resolution Survey Spectrometer (MRSS) for the Origins Space Telescope: Enabling 3-D Surveys of the Universe in the Far-IR. In American Astronomical Society Meeting Abstracts, January 2018.
[CG02] M. R. Calabretta and E. W. Greisen. Representations of celestial coordinates in FITS. Astronomy and Astrophysics, 395:10771122, December 2002. arXiv:astro-ph/0207413, doi: 10.1051/0004-6361:20021327.

[DSB ${ }^{+}$16] Richard Dekany, Roger M. Smith, Justin Belicki, Alexandre Delacroix, Gina Duggan, Michael Feeney, David Hale, Stephen Kaye, Jennifer Milburn, Patrick Murphy, Michael Porter, Daniel J. Reiley, Reed L. Riddle, Hector Rodriguez, and Eric C. Bellm. The Zwicky Transient Facility Camera. In Ground-based and Airborne Instrumentation for Astronomy VI, volume 9908, page 99085M, August 2016. doi:10.1117/12.2234558.

$\left[\mathrm{GBV}^{+} 16\right]$ Gaia Collaboration, A. G. A. Brown, A. Vallenari, T. Prusti, J. H. J. de Bruijne, F. Mignard, R. Drimmel, C. Babusiaux, C. A. L. Bailer-Jones, U. Bastian, and et al. Gaia Data Release 1. Summary of the astrometric, photometric, and survey properties. Astronomy and Astrophysics, 595:A2, November 2016. arXiv: 1609.04172, doi:10.1051/0004-6361/201629512.

[GPd $\left.{ }^{+} 16\right]$ Gaia Collaboration, T. Prusti, J. H. J. de Bruijne, A. G. A. Brown, A. Vallenari, C. Babusiaux, C. A. L. Bailer-Jones, U. Bastian, M. Biermann, D. W. Evans, and et al. The Gaia mission. Astronomy and Astrophysics, 595:A1, November 2016. arXiv: 1609.04153, doi:10.1051/0004-6361/201629272.

$\left[\mathrm{GZT}^{+} 18\right]$ N. J. Goldbaum, J. A. ZuHone, M. J. Turk, K. Kowalik, and A. L. Rosen. unyt: Handle, manipulate, and convert data with units in Python. ArXiv e-prints, June 2018. arXiv:1806.02417.

$\left[\mathrm{LKD}^{+} 09\right]$ Nicholas M. Law, Shrinivas R. Kulkarni, Richard G. Dekany, Eran O. Ofek, Robert M. Quimby, Peter E. Nugent, Jason Surace, Carl C. Grillmair, Joshua S. Bloom, Mansi M. Kasliwal, Lars 
Bildsten, Tim Brown, S. Bradley Cenko, David Ciardi, Ernest Croner, S. George Djorgovski, Julian van Eyken, Alexei V. Filippenko, Derek B. Fox, Avishay Gal- Yam, David Hale, Nouhad Hamam, George Helou, John Henning, D. Andrew Howell, Janet Jacobsen, Russ Laher, Sean Mattingly, Dan McKenna, Andrew Pickles, Dovi Poznanski, Gustavo Rahmer, Arne Rau, Wayne Rosing, Michael Shara, Roger Smith, Dan Starr, Mark Sullivan, Viswa Velur, Richard Walters, and Jeff Zolkower. The Palomar Transient Factory: System Overview, Performance, and First Results. Publications of the Astronomical Society of the Pacific, 121:1395, December 2009. arXiv:0906.5350, doi:10.1086/648598.

$\left[\mathrm{LMG}^{+} 18\right]$ Russ R. Laher, Frank J. Masci, Steve Groom, Benjamin Rusholme, David L. Shupe, Ed Jackson, Jason Surace, Dave Flynn, Walter Landry, Scott Terek, George Helou, Ron Beck, Eugean Hacopians, Umaa Rebbapragada, Brian Bue, Roger M. Smith, Richard G. Dekany, Adam A. Miller, S. B. Cenko, Eric Bellm, Maria Patterson, Thomas Kupfer, Lin Yan, Tom Barlow, Matthew Graham, Mansi M. Kasliwal, Thomas A. Prince, and Shrinivas R. Kulkarni. Processing Images from the Zwicky Transient Facility. RTSE Conference Proceedings, 2018. arXiv: 1708.01584

[PAA $\left.{ }^{+} 16\right]$ Planck Collaboration, P. A. R. Ade, N. Aghanim, M. Arnaud, M. Ashdown, J. Aumont, C. Baccigalupi, A. J. Banday, R. B. Barreiro, J. G. Bartlett, N. Bartolo, E. Battaner, R. Battye, K. Benabed, A. Benoît, A. Benoit-Lévy, J. P. Bernard, M. Bersanelli, P. Bielewicz, J. J. Bock, A. Bonaldi, L. Bonavera, J. R. Bond, J. Borrill, F. R. Bouchet, F. Boulanger, M. Bucher, C. Burigana, R. C. Butler, E. Calabrese, J. F. Cardoso, A. Catalano, A. Challinor, A. Chamballu, R. R. Chary, H. C. Chiang, J. Chluba, P. R. Christensen, S. Church, D. L. Clements, S. Colombi, L. P. L. Colombo, C. Combet, A. Coulais, B. P. Crill, A. Curto, F. Cuttaia, L. Danese, R. D. Davies, R. J. Davis, P. de Bernardis, A. de Rosa, G. de Zotti, J. Delabrouille, F. X. Désert, E. Di Valentino, C. Dickinson, J. M. Diego, K. Dolag, H. Dole, S. Donzelli, O. Doré, M. Douspis, A. Ducout, J. Dunkley, X. Dupac, G. Efstathiou, F. Elsner, T. A. Enßlin, H. K. Eriksen, M. Farhang, J. Fergusson, F. Finelli, O. Forni, M. Frailis, A. A. Fraisse, E. Franceschi, A. Frejsel, S. Galeotta, S. Galli, K. Ganga, C. Gauthier, M. Gerbino, T. Ghosh, M. Giard, Y. Giraud-Héraud, E. Giusarma, E. Gjerløw, J. González-Nuevo, K. M. Górski, S. Gratton, A. Gregorio, A. Gruppuso, J. E. Gudmundsson, J. Hamann, F. K. Hansen, D. Hanson, D. L. Harrison, G. Helou, S. Henrot- Versillé, C. Hernández-Monteagudo, D. Herranz, S. R. Hildebrandt, E. Hivon, M. Hobson, W. A. Holmes, A. Hornstrup, W. Hovest, Z. Huang, K. M. Huffenberger, G. Hurier, A. H. Jaffe, T. R. Jaffe, W. C. Jones, M. Juvela, E. Keihänen, R. Keskitalo, T. S. Kisner, R. Kneissl, J. Knoche, L. Knox, M. Kunz, H. KurkiSuonio, G. Lagache, A. Lähteenmäki, J. M. Lamarre, A. Lasenby, M. Lattanzi, C. R. Lawrence, J. P. Leahy, R. Leonardi, J. Lesgourgues, F. Levrier, A. Lewis, M. Liguori, P. B. Lilje, M. LindenVørnle, M. López-Caniego, P. M. Lubin, J. F. Macías-Pérez, G. Maggio, D. Maino, N. Mandolesi, A. Mangilli, A. Marchini, M. Maris, P. G. Martin, M. Martinelli, E. Martínez-González, S. Masi, S. Matarrese, P. McGehee, P. R. Meinhold, A. Melchiorri, J. B. Melin, L. Mendes, A. Mennella, M. Migliaccio, M. Millea, S. Mitra, M. A. Miville-Deschênes, A. Moneti, L. Montier, G. Morgante, D. Mortlock, A. Moss, D. Munshi, J. A. Murphy, P. Naselsky, F. Nati, P. Natoli, C. B. Netterfield, H. U. NørgaardNielsen, F. Noviello, D. Novikov, I. Novikov, C. A. Oxborrow, F. Paci, L. Pagano, F. Pajot, R. Paladini, D. Paoletti, B. Partridge, F. Pasian, G. Patanchon, T. J. Pearson, O. Perdereau, L. Perotto, F. Perrotta, V. Pettorino, F. Piacentini, M. Piat, E. Pierpaoli, D. Pietrobon, S. Plaszczynski, E. Pointecouteau, G. Polenta, L. Popa, G. W. Pratt, G. Prézeau, S. Prunet, J. L. Puget, J. P. Rachen, W. T. Reach, R. Rebolo, M. Reinecke, M. Remazeilles, C. Renault, A. Renzi, I. Ristorcelli, G. Rocha, C. Rosset, M. Rossetti, G. Roudier, B. Rouillé d'Orfeuil, M. Rowan-Robinson, J. A. Rubiño-Martín, B. Rusholme, N. Said, V. Salvatelli, L. Salvati, M. Sandri, D. Santos, M. Savelainen, G. Savini, D. Scott, M. D. Seiffert, P. Serra, E. P. S. Shellard, L. D. Spencer, M. Spinelli, V. Stolyarov, R. Stompor, R. Sudiwala, R. Sunyaev, D. Sutton, A. S. Suur-Uski, J. F. Sygnet, J. A. Tauber, L. Terenzi, L. Toffolatti, M. Tomasi, M. Tristram, T. Trombetti, M. Tucci, J. Tuovinen, M. Türler, G. Umana, L. Valenziano, J. Valiviita, F. Van Tent, P. Vielva, F. Villa, L. A. Wade, B. D. Wandelt, I. K. Wehus, M. White, S. D. M. White, A. Wilkinson, D. Yvon, A. Zacchei, and A. Zonca. Planck 2015 results. XIII. Cosmological param- eters. Astronomy and Astrophysics, 594:A13, September 2016. doi:10.1051/0004-6361/201525830.

[STS13] STScI Development Team. pysynphot: Synthetic photometry software package. Astrophysics Source Code Library, March 2013. arXiv:1303.023.

[TPS $\left.{ }^{+} 18\right]$ The Astropy Collaboration, A. M. Price-Whelan, B. M. Sipócz, H. M. Günther, P. L. Lim, S. M. Crawford, S. Conseil, D. L. Shupe, M. W. Craig, N. Dencheva, A. Ginsburg, J. T. VanderPlas, L. D. Bradley, D. Pérez- Suárez, M. de Val-Borro, T. L. Aldcroft, K. L. Cruz, T. P. Robitaille, E. J. Tollerud, C. Ardelean, T. Babej, M. Bachetti, A. V. Bakanov, S. P. Bamford, G. Barentsen, P. Barmby, A. Baumbach, K. L. Berry, F. Biscani, M. Boquien, K. A. Bostroem, L. G. Bouma, G. B. Brammer, E. M. Bray, H. Breytenbach, H. Buddelmeijer, D. J. Burke, G. Calderone, J. L. Cano Rodríguez, M. Cara, J. V. M. Cardoso, S. Cheedella, Y. Copin, D. Crichton, D. DÁvella, C. Deil, É. Depagne, J. P. Dietrich, A. Donath, M. Droettboom, N. Earl, T. Erben, S. Fabbro, L. A. Ferreira, T. Finethy, R. T. Fox, L. H. Garrison, S. L. J. Gibbons, D. A. Goldstein, R. Gommers, J. P. Greco, P. Greenfield, A. M. Groener, F. Grollier, A. Hagen, P. Hirst, D. Homeier, A. J. Horton, G. Hosseinzadeh, L. Hu, J. S. Hunkeler, Ž. Ivezić, A. Jain, T. Jenness, G. Kanarek, S. Kendrew, N. S. Kern, W. E. Kerzendorf, A. Khvalko, J. King, D. Kirkby, A. M. Kulkarni, A. Kumar, A. Lee, D. Lenz, S. P. Littlefair, Z. Ma, D. M. Macleod, M. Mastropietro, C. McCully, S. Montagnac, B. M. Morris, M. Mueller, S. J. Mumford, D. Muna, N. A. Murphy, S. Nelson, G. H. Nguyen, J. P. Ninan, M. Nöthe, S. Ogaz, S. Oh, J. K. Parejko, N. Parley, S. Pascual, R. Patil, A. A. Patil, A. L. Plunkett, J. X. Prochaska, T. Rastogi, V. Reddy Janga, J. Sabater, P. Sakurikar, M. Seifert, L. E. Sherbert, H. Sherwood-Taylor, A. Y. Shih, J. Sick, M. T. Silbiger, S. Singanamalla, L. P. Singer, P. H. Sladen, K. A. Sooley, S. Sornarajah, O. Streicher, P. Teuben, S. W. Thomas, G. R. Tremblay, J. E. H. Turner, V. Terrón, M. H. van Kerkwijk, A. de la Vega, L. L. Watkins, B. A. Weaver, J. B. Whitmore, J. Woillez, and V. Zabalza. The Astropy Project: Building an inclusive, open-science project and status of the v2.0 core package. ArXiv e-prints, page arXiv:1801.02634, January 2018. arXiv: 1801.02634 . 